\title{
Signal Source Localization with Tetrodes: Experimental Verification
}

\author{
Chang Won Lee, Christine E. King, Shun Chi Wu, A. Lee Swindlehurst, and Zoran Nenadic
}

\begin{abstract}
Multi-sensor electrodes for extracellular recording of neuronal action potentials have significantly increased the signal-to-noise ratio (SNR) in neurophysiological experiments, ultimately leading to a more accurate interpretation of scientific data. Apart from improving SNR, we hypothesize that these electrodes can be used to estimate the location of underlying neuronal signal sources, and perhaps other parameters such as the size and shape of neurons whose activities are being recorded. This study introduces the multiple signal classification (MUSIC) algorithm to the problem of neuron localization and presents the first experimental demonstration of signal source localization using commercially available 4-sensor electrodes (tetrodes).
\end{abstract}

\section{INTRODUCTION}

Extracellular recording of action potentials (APs) from one or more neurons has become a fundamental technique for in vivo neurophysiology studies, especially those involving behaving animals [1] and humans [2], where direct intracellular measurements of APs may be impractical or impossible. With the advent of multi-sensor recording electrodes, such as stereotrodes [3] and tetrodes [4], the signal-to-noise ratio (SNR) of extracellular recordings has been significantly improved. This facilitates more accurate signal analysis, such as AP detection [5], [6] and classification [7], [8], and in turn a more accurate interpretation of scientific data.

The typical tetrode layout consists of 4 sensors $\sim 50$ $\mu \mathrm{m}$ apart, which can be achieved by twisting a bundle of 4 microwires [4], coating 4 independent platinum-tungsten cores with glass [9] (see Fig. 1), or micro-machining planar silicon arrays with iridium recording sites [10]. In addition to improving SNR, tetrodes have been used for identification of bursting neurons [4], estimation of voltage decay constants [4], and establishing the correspondence of APs over multiple recording sessions [9]. Less traditional uses of tetrodes include localization of AP sources [11] and estimation of the flow of electric charges during APs [12].

In this article we introduce a novel technique for localization of neurons based on a multiple signal classification (MUSIC) algorithm. While originally developed for estimation of parameters in antenna arrays [13], MUSIC has been successfully employed in biomedical applications

This work was supported in part by the National Science Foundation under Grant 1056105.

C. W. Lee and C. E. King are with the Department of Biomedical Engineering, University of California, Irvine, CA 92697, USA cwlee@uci.edu, kingce@uci.edu

S.-C. Wu and A. L. Swindlehurst are with the Department of Electrical Engineering and Computer Science, University of California, Irvine, CA 92697, USA scwu@uci.edu, swindle@uci.edu

Z. Nenadic is with the Departments of Biomedical Engineering, and Electrical Engineering and Computer Science, University of California, Irvine, CA 92697, USA znenadic@uci.edu

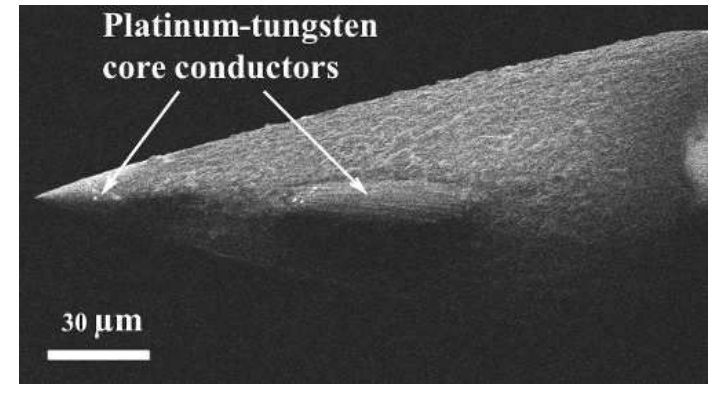

Fig. 1. Scanning electron microscope image of tetrode (Thomas Recording, Giessen, Germany). Two platinum-tungsten sensors are visible from this perspective.

such as localization of electroencephalogram and magnetoencephalogram signal sources [14]. Motivated by these applications, we hypothesize that the MUSIC algorithm can also be applied to the localization of neurons based on their multi-sensor AP measurements. In addition, our study provides an experimental validation of this technique with a commercial tetrode device placed in proximity to a dipolelike stimulator. In particular, we show that the estimated source lies within $\sim 40 \mu \mathrm{m}$ of the true signal source. By factoring in the heterogeneity of the conductive medium, the localization error can be further reduced to $<3 \mu \mathrm{m}$.

\section{BACKGROUND AND SIGNIFICANCE}

The first experimental study concerned with localization of neurons, often referred to as source localization, was reported by Csicsvari et al. [15], who recorded extracellular APs using two-dimensional (2D) silicon arrays. The vertical position of neurons was estimated as the depth of a sensor with the largest AP amplitude. In a related study, Bartho et al. [16] estimated the position of active neurons as a weighted combination of the locations of recording sensors. These approaches, however, are largely heuristic and make no attempt to estimate neurons' full 3D locations.

Chelaru and Jog [17] developed a deterministic source localization algorithm for microwire tetrodes based on a simple forward model. By numerically inverting the model, they reported on the ability to localize three distinct neurons, whose activities were simultaneously recorded with a single tetrode. However, this study as well as those in [15] and [16] were performed in vivo, where the actual positions of neurons were unknown, and so these methods lack validation. Similarly, Somogyvari et al. [18] developed a model-based source localization technique suitable for linear electrode arrays, but the 3D location of the source could not be estimated due to the linear arrangement of the sensors. 
Inspired by the study in [17], we demonstrated that the inversion of a simple monopole forward model

$$
\phi_{i}(t)=\frac{I_{m}(t)}{4 \pi \sigma r_{i}(x, y, z)} \quad i=1,2,3,4,
$$

admits a closed-form solution [19], i.e. the position $(x, y, z)$ of the neuronal source can be found analytically. In the above model, $\phi_{i}$ represents the extracellular potential at sensor $i, I_{m}$ is the time-dependent membrane current, $\sigma$ is the conductivity per unit length of extracellular space, and $r_{i}(x, y, z)$ is the distance between the sensor $i$ and the source. Fig. 2 illustrates an application of this technique to source localization in a two-neuron computational model, where individual neurons were modeled using the compartmental approach [20]. Note, however, that the inversion of (1) is not guaranteed, especially if there is significant mismatch between $\phi_{i}$ predicted by the monopole model and simulated potentials, $\psi_{i}[19]$.

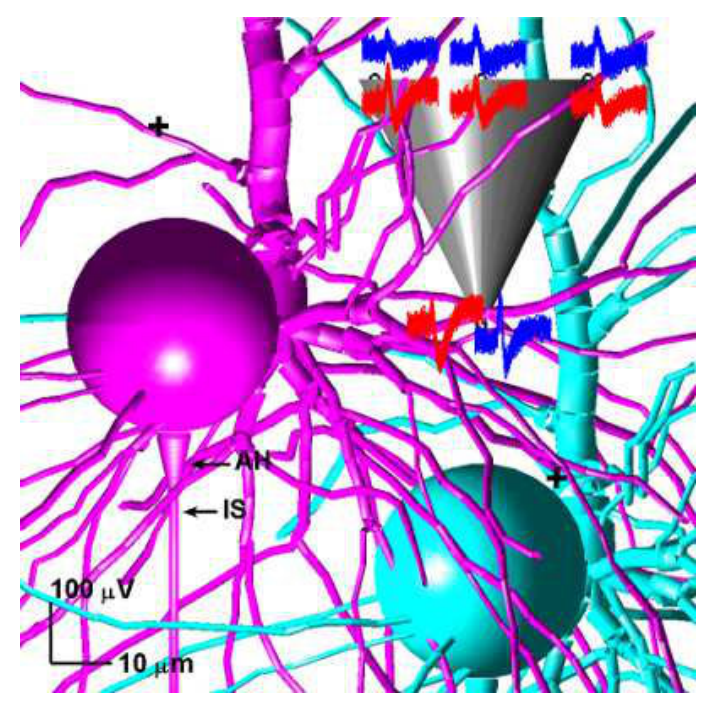

Fig. 2. Simulation of 50 noisy APs (duration $2 \mathrm{msec}$ ) sensed by a tetrode(gray cone) with sensors marked by $\circ$. Red and blue traces correspond to the cells on the left and bottom right, respectively. The cylindrical compartments are dendrites, the axon hillock ( $\mathrm{AH})$, and the initial segment (IS), and the spherical compartment is the soma. Estimated source locations are marked by + .

The ability to localize neurons whose APs are being recorded may significantly alleviate the tedious process of guidance of multi-sensor electrodes often employed in acute recording experiments. Furthermore, by performing source localization over time, migration trends of neurons may be estimated, which may further improve these experimental procedures. In chronic recording experiments, the ability to track neurons' positions over time may help distinguish changes in recorded signals due to these tissue drifts from those due to other factors such as neuronal plasticity and adaptation, as well as scar tissue formation and reactive gliosis. In summary, the ability to localize neurons based on their extracellularly recorded APs may have a profound impact on the way recording experiments are performed in both acute and chronic conditions.

\section{MATERIALS AND METHODS}

The MUSIC algorithm [13] offers an alternative solution to the source localization problem. It assumes that multisensor measurements, $\Psi \in \mathbb{R}^{m \times 1}$, of a signal originating from a single source can be modeled as

$$
\Psi(t)=\mathbf{a}(\mathbf{r}) s(t)+\mathbf{w}(t),
$$

where $m$ is the number of sensors, $\mathbf{a} \in \mathbb{R}^{m \times 1}$ is the socalled lead field vector (LFV), $\mathbf{r}=[x, y, z]^{\mathrm{T}}$ is the unknown location of the source, $s \in \mathbb{R}^{1 \times 1}$ is the source signal, and $\mathbf{w} \in \mathbb{R}^{m \times 1}$ is additive noise. In the case of neuronal source localization with a tetrode $(m=4)$, the elements of a are $a_{i}=1 /\left(4 \pi \sigma r_{i}(\mathbf{r})\right), i=1,2,3,4$, while the source signal is the membrane current, $I_{m}$ [compare (1) and (2)]. However, unlike the framework presented in [19] that seeks the exact inversion of the model (1), MUSIC utilizes an optimization framework, and is therefore less susceptible to the mismatch between measurements $\Psi$ and model predictions. More specifically, MUSIC seeks the source location whose LFV is most orthogonal to the noise subspace $\mathbf{E}_{N}$, i.e.

$$
\mathbf{r}^{\star}=\arg \min _{\mathbf{r}} \frac{\mathbf{a}^{\mathrm{T}}(\mathbf{r}) \mathbf{E}_{N} \mathbf{E}_{N}^{\mathrm{T}} \mathbf{a}(\mathbf{r})}{\mathbf{a}^{\mathrm{T}}(\mathbf{r}) \mathbf{a}(\mathbf{r})},
$$

where $\mathbf{E}_{N} \in \mathbb{R}^{4 \times 3}$ can be found by the singular value decomposition of $\boldsymbol{\Psi} \in \mathbb{R}^{4 \times n}$. Assuming the number of time samples $n>4$, the columns of $\mathbf{E}_{N}$ can be chosen as the left singular eigenvectors of $\boldsymbol{\Psi}$ corresponding to the smallest three singular values.

Experimental setup consisted of an inverted microscope (Olympus IX51, Olympus America, Center Valley, PA), a tetrode motorized microdrive system (Thomas Recording, Giessen, Germany), and a data acquisition system (TuckerDavis Technologies, Alachua, FL). In addition, an auxiliary system (MP150, Biopac Systems, Goleta, CA) was connected to a single-sensor microelectrode (Alpha Omega Co. USA, Alpharetta, GA), which was used as a stimulator. Both the tetrode microdrive (Mini Matrix, Thomas Recording, Giessen, Germany) and the stimulating electrode were mounted on a microscope-compatible micromanipulator (Narishige International USA, East Meadow, NY). The stimulating electrode was placed in the center of a Petri dish filled with $0.9 \%$ saline solution, with a reference electrode that consisted of a wire placed against the Petri dish wall along its circumference. While technically a dipole, the electric field of this stimulator, at least locally around the stimulating electrode tip, is consistent with a monopole. The tetrode was positioned near the tip of the stimulating electrode using a combination of coarse (micromanipulator) and fine (microdrive) adjustments under the microscope guidance. The stimulator was programmed using AcqKnowledge software (Biopac Systems, Goleta, CA). All experiments were performed at a room temperature $\left(20^{\circ} \mathrm{C}\right)$.

\section{RESULTS AND DISCUSSION}

\section{A. Results}

To facilitate accurate position measurements, the focal plane of the microscope was adjusted until the tip of the 


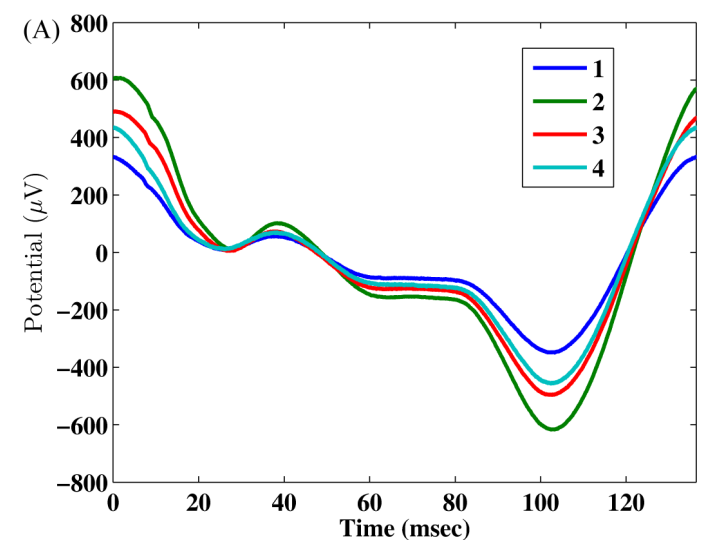

Fig. 3. (A) Average signals $(N=50)$ recorded by the sensor at the tetrode tip.

stimulating electrode was clearly visible. The tetrode (Fig. 1) was then placed in the vicinity of the stimulating electrode and its position was fine-tuned using computer control of the motorized microdrive. The adjustment was applied until the tetrode tip reached the focal plane. Therefore, throughout this section, we will assume that the depths ("z-coordinates") of two tips are the same. Prior to the experiment, the impedances of the four sensors were measured at $1 \mathrm{kHz}$ with an impedance monitor (Bak Electronics, Mount Airy, MD) and were found to be in the range 0.5-0.9 $\mathrm{M} \Omega$.

Using AcqKnowledge software, a stimulus pattern was programmed as a sine wave with a frequency of $7 \mathrm{~Hz}$. Note that this frequency is not a subharmonic of the power line noise $(60 \mathrm{~Hz})$, and therefore the signals were unambiguously detectable at the tetrode sensors. The pattern was delivered to the stimulating electrode by utilizing the MP150 system's analog output channel, with the signal amplitude set to $1 \mathrm{~V}$. To ensure statistically meaningful results, approximately 7 seconds of data were recorded ( $\sim 50$ cycles). The data were amplified, digitized (sampling rate: $25 \mathrm{kHz}$, resolution: 16 bits), and saved for further analysis. The above procedure was then repeated by changing the position of the tetrode with the micromanipulator.

Fig. 3(A) shows the recorded stimulation patterns averaged over 50 cycles. The individual traces represent signals recorded by the sensor located at the tip as the tetrode's position was varied in the vicinity of the stimulating electrode (see Fig. 4). As predicted by the model (2), the strongest signal is recorded at position 2 , where the distance between the sensor and the source (the tip of the stimulating electrode) is smallest $(\sim 50 \mu \mathrm{m})$. Conversely, the signal is the weakest at position 1, which is $\sim 100 \mu \mathrm{m}$ away from the source. By supplying these signals to the source localization algorithm (3), the estimated location of the source is found to be: $\mathbf{r}^{\star}=(9.8,-39.7,-0.11) \mu \mathrm{m}$, with the origin defined at the source location and axes defined as illustrated in Fig. 4. It can also be seen in Fig. 4 that the estimated source location is $\sim 40 \mu \mathrm{m}$ away from the true source location.

Such a relatively large bias indicates that the model proposed by (2) is not accurate. To circumvent this problem, we redefined the LFV such that $\bar{a}_{i}=1 /\left(4 \pi \sigma_{i} r_{i}(\mathbf{r})\right)$, where

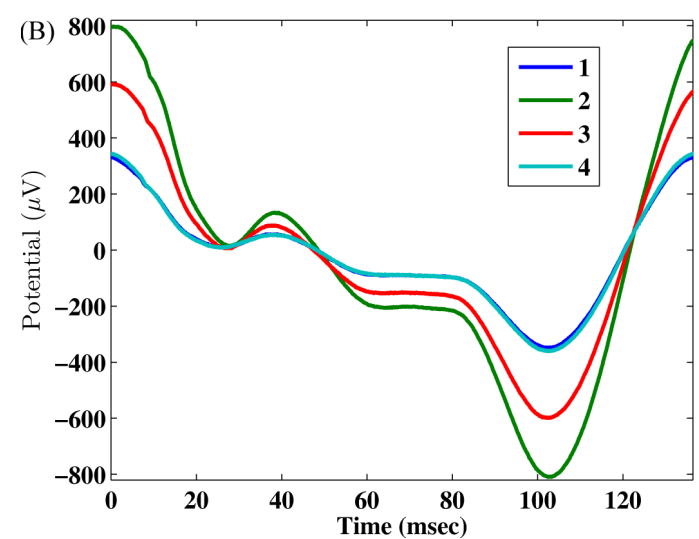

(B) The same signals scaled to compensate for inhomogeneous conductivity.

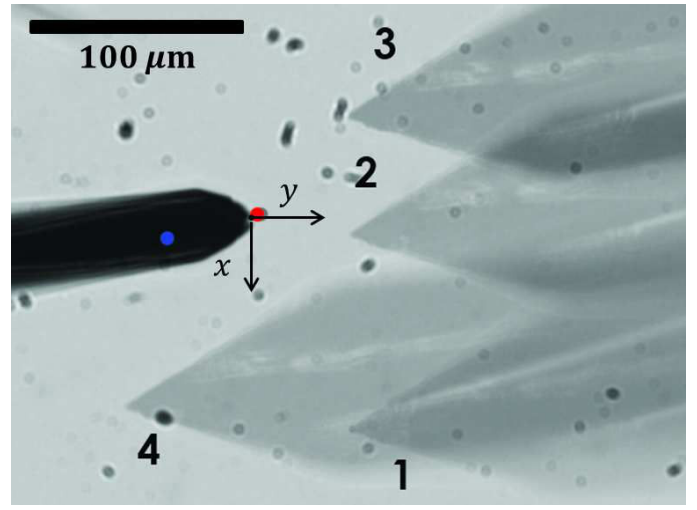

Fig. 4. Superposition of microscopy images showing four tetrode positions (marked by 1-4). On the left is the stimulating electrode with the source defined at its tip. Blue and red dots respectively mark the estimated source location before and after accounting for the heterogeneity of the medium.

at each position we have: $\sigma_{i}=k_{i} \sigma$, with $\sigma$ representing some baseline value. By assuming $k_{1}=1$, it readily follows that: $k_{i} \approx\left(\psi_{1}(t) r_{1}\right) /\left(\psi_{i}(t) r_{i}\right), i=2,3,4$. While $k_{i}$ is a time-dependent quantity, we have shown in [21] that its value remains stable as long as $\psi_{i}(t) \neq 0$. After elementary calculations, we obtained: $k_{2}=1.31, k_{3}=1.21$, and $k_{4}=0.79$. This allows the model (2) to be redefined as:

$$
\bar{\Psi}(t)=\mathbf{a}(\mathbf{r}) s(t)+\overline{\mathbf{w}}(t),
$$

where $\bar{\psi}_{i}(t)=k_{i} \psi_{i}(t), i=1,2,3,4$, represent the scaling of the original measurements. Fig. 3(B) shows the average patterns after scaling. Note that the signal at position 1 remains unchanged $\left(k_{1}=1\right)$. By estimating the location of the source (3) with these scaled data, we obtained: $\mathbf{r}^{\star}=$ $(-0.5,2.9,-0.05) \mu \mathrm{m}$. The estimated source location is also shown in Fig. 4 and is $<3 \mu \mathrm{m}$ away from the source location.

\section{B. Discussion}

The proposed algorithm was validated using only the sensor at the tetrode tip, and the "virtual tetrode" was formed by placing the tetrode at 4 different locations. This scenario is not applicable in experimental practice since tetrodes primarily move in a linear fashion. The main reason for 
adopting this approach, as opposed to utilizing all sensors at a single tetrode location, is that the sensors in general cannot be visualized under the microscope (with the exception of the tip), and so their exact locations remain unknown. Based on manufacturer's data and the knowledge of the tip location, constraints can be placed on the location of the remaining sensors. MUSIC can then be used to estimate the locations of both the source and the remaining sensors, however, the lack of the ground truth would confound the validation process.

By assuming homogeneous isotropic medium, i.e. $\sigma=$ const., the ratio of the signals in Fig. 3(A) is determined by the ratio of the distances between the tetrode tip and the source. However, by comparing the signals at positions 1 and 4 (both positions are $\sim 100 \mu \mathrm{m}$ from the source), it is apparent that the homogeneity assumption must be violated. Note that the choice $k_{1}=1$ is arbitrary and must be interpreted in the context of $k_{2}, k_{3}$ and $k_{4}$. Based on the obtained values, it follows that the conductivity between the source and position 2 is the highest, while that between the source and position 4 is the lowest. Finally, note that in addition to inhomogeneity, these differences could be explained by directional sensitivity (anisotropy).

While the proposed method has been developed and experimentally validated on a single source [c.f. (2)], it readily extends to multiple sources such as dipoles. This is significant since there are neurons, such as layer- 4 pyramidal cells, whose elongated dendritic trees generate electric fields that are reminiscent of dipoles [22]. On the other hand, the electric fields of radially symmetric neurons, such as stellate cells, tend to be closed and thus are consistent with monopoles. Therefore, in a more general framework, in addition to localizing neuronal sources, multi-sensor measurements of APs may be used for estimating the shape of a neuron whose activity is being recorded.

\section{CONCLUSIONS AND FUTURE WORK}

We have introduced a neuron localization scheme based on the MUSIC algorithm that utilizes multi-sensor extracellular AP measurements. While the method was developed for tetrodes, it readily extends to other types of multi-sensor arrays, and can be adapted to other types of generative models such as dipoles. We have also presented the first experimental validation of this technique using a commercial tetrode device. Based on the experimental results, we conclude that the localization of neuronal sources with tetrodes is feasible, although the estimated source location may be biased due to the heterogeneity and anisotropy of the extracellular medium.

Our future research efforts will be directed toward in vitro validation of the proposed technique on neural systems. This, for example, may be achieved by utilizing cortical brain slices, where specific neurons can be directly stimulated by a micropipette to elicit APs. Using tetrodes and other types of multi-sensor arrays [10] to record from such neurons, valuable data will be collected. In addition to validating the positions and, perhaps, the shapes of these neurons, other parameters such as the intensity (peak membrane current) of the source may be estimated. Since smaller neurons generate smaller membrane currents [23], the estimated source intensity may be used to estimate the size of the neuron.

\section{REFERENCES}

[1] G. Buzsaki. Large-scale recording of neuronal ensembles. Nat Neurosci, 7(5):446-451, 2004.

[2] R.Q. Quiroga, L. Reddy, G. Kreiman, C. Koch, and I. Fried. Invariant visual representation by single neurons in the human brain. Nature, 435(7045):1102-1107, 2005.

[3] B.L. McNaughton, J. O'Keefe, and C.A. Barnes. The stereotrode: a new technique for simultaneous isolation of several single units in the central nervous system from multiple unit records. J Neurosci Methods, 8(4):391-397, 1983.

[4] C.M. Gray, P.E. Maldonado, M. Wilson, and B. McNaughton. Tetrodes markedly improve the reliability and yield of multiple single-unit isolation from multi-unit recordings in cat striate cortex. $J$ Neurosci Methods, 63(1-2):43-54, 1995.

[5] Z. Nenadic and J.W. Burdick. Spike detection using the continuous wavelet transform. IEEE Trans Biomed Eng, 52(1):74-87, 2005.

[6] R. Benitez and Z. Nenadic. Robust unsupervised detection of action potentials with probabilistic models. IEEE Trans. Biomed. Eng., 55(4):1344-1354, 2008.

[7] M.S. Lewicki. A review of methods for spike sorting: the detection and classification of neural action potentials. Network, 9(4):R53-R78, 1998.

[8] R.Q. Quiroga, Z. Nadasdy, and Y. Ben-Shaul. Unsupervised spike detection and sorting with wavelets and superparamagnetic clustering. Neural Comput, 16(8):1661-1687, 2004.

[9] A.A. Emondi, S.P. Rebrik, A.V. Kurgansky, and K.D. Miller. Tracking neurons recorded from tetrodes across time. J Neurosci Methods, 135(1-2):95-105, 2004.

[10] K.L. Drake, K.D. Wise, J. Farraye, D.J. Anderson, and S.L. BeMent. Performance of planar multisite microprobes in recording extracellular single-unit intracortical activity. IEEE Trans Biomed Eng, 35(9):719$732,1988$.

[11] M.S. Jog, C.I. Connolly, Y. Kubota, D.R. Iyengar, L. Garrido, R. Harlan, and A.M. Graybiel. Tetrode technology: advances in implantable hardware, neuroimaging, and data analysis techniques. J Neurosci Methods, 117(2):141-152, 2002.

[12] D. Aur, C.I. Connolly, and M.S. Jog. Computing spike directivity with tetrodes. J Neurosci Methods, 149(1):57-63, 2005.

[13] R. Schmidt. Multiple emitter location and signal parameter estimation. IEEE Trans. Antennas Propagat., 34(3):276-280, 1986.

[14] J.C. Mosher and R.M. Leahy. Recursive MUSIC: A framework for EEG and MEG source localization. IEEE Trans. Biomed. Eng., 45(11):1342-1354, 1998.

[15] J. Csicsvari, D.A. Henze, B. Jamieson, K.D. Harris, A. Sirota, P. Bartho, K.D. Wise, and G. Buzsaki. Massively parallel recording of unit and local field potentials with silicon-based electrodes. $J$ Neurophysiol, 90(2):1314-1323, 2003.

[16] P. Bartho, H. Hirase, L. Monconduit, M. Zugaro, K.D. Harris, and G. Buzsaki. Characterization of neocortical principal cells and interneurons by network interactions and extracellular features. $J$ Neurophysiol, 92(1):600-608, 2004.

[17] M.I. Chelaru and M.S. Jog. Spike source localization with tetrodes. $J$ Neurosci Methods, 142(2):305-315, 2005.

[18] Z. Somogyvari, L. Zalanyi, I. Ulbert, and P. Erdi. Model-based source localization of extracellular action potentials. J Neurosci Methods, 147(2):126-137, 2005.

[19] C.W. Lee, H. Dang, and Z. Nenadic. An efficient algorithm for current source localization with tetrodes. In Proceedings of the 29th Annual International Conference of the IEEE Engineering in Medicine and Biology Society, pages 1282-1285, 2007.

[20] Z.F. Mainen and T.J. Sejnowski. Influence of dendritic structure on firing pattern in model neocortical neurons. Nature, 382(6589):363366, 1996.

[21] C.W. Lee, S.-C. Wu, A.L. Swindlehurst, and Z. Nenadic. Experimental verification of source localization with tetrodes. In Soc. Neurosci. Abstr. 40: 820.7, 2010.

[22] W. Rall. Electrophysiology of a dendritic neuron model. Biophys $J$, 2(2 Pt 2):145-167, 1962.

[23] D.R. Humphrey. Electrophysiological Techniques. Society for Neuroscience, Atlanta, 1979. 